\title{
New Diagnostic Methods for Tuberculosis
}

\author{
Melissa R. Nyendak, MD, MHS [Assistant Professor of Medicine] \\ Division of Infectious Diseases Oregon Health and Science University 3181 SW Sam Jackson \\ Park Road L457 Portland, Oregon 97239 (503) 418-1811 nyendakm@ohsu.edu \\ Deborah A. Lewinsohn, MD [Associate Professor of Medicine] \\ Division of Pediatric Infectious Diseases Oregon Health and Science University 3181 SW Sam \\ Jackson Park Road CDRCP-2207 Portland, Oregon 97239 (503) 494-0950 lewinsde@ohsu.edu \\ David M. Lewinsohn, MD, PhD [Associate Professor Medicine] \\ Division of Pulmonary and Critical Care Medicine Portland VA Medical Center, Pulmonary \& CCM \\ R\&D 11, PVAMC 3710 SW US Veterans Road Portland, Oregon 97239
}

\section{Abstract}

Purpose of Review-During the past decade, laboratory tests for the detection of Mycobacterium tuberculosis (Mtb) have improved dramatically. Improvements in the ability to detect latent infection with Mtb, disease associated with Mtb, and strains resistant to commonly used antibiotics are reviewed.

Recent Findings-Advances in the detection of Mtb include LED fluorescence microscopy, nucleic acid amplification of Mtb and drug resistant strains, and more rapid liquid culture with adjunct drug susceptibility testing. In the detection of latent tuberculosis (LTBI) interferon gamma release assays (IGRAs) offer improved accuracy over the tuberculin skin test (TST).

Summary-The last 10 years has seen the most rapid growth in new diagnostics for Mtb in over a century. While these tests offer improvements in the ability to detect Mtb, drug resistant isolates, and those with LTBI, these improvements are counter-balanced by the need to deploy these tests in areas where Mtb burden is highest.

\section{Keywords}

Tuberculosis; Diagnosis; Molecular Probes; Immunological Tests

\section{Introduction}

The evolutionary success of Mycobacterium tuberculosis (Mtb) is reflected in its coexistence with humans for over two millennia. Current estimates by the World Health Organization report over 9 million new cases and 1.7 million deaths in 2006 (1). The economic impact of tuberculosis on those in the prime of their lives is profound (2). Because tuberculosis is a chronic illness with lengthy periods of potential transmission, improved case finding could have a profound impact (3).

Until recently, the pace of new, relevant diagnostics had not kept pace with the global emergency. However, within the last ten years, advances in the molecular biology of Mtb in conjunction with modern immunological methods have led to improvement in our ability to detect Mtb and to delineate the host's response to infection. It is with these new tools that the 
WHO has proclaimed an ambitious target of detecting $70 \%$ of sputum smear positive cases and to successfully treat $85 \%(1)$. The interplay between the host response, bacterial burden, and correlates of protection/disease are shown in figure 1, highlighting the diagnostic potential across the spectrum of Mtb disease. In this review, we discuss the new diagnostic techniques and the current evidence behind their use.

\section{Advances in Microscopy}

Smear microscopy with carbol fuchsin and flourochromes such as auramine-rhodamine remains a mainstay in the detection of Mtb in clinical specimens and widely supported by the WHO (4). Fluorescence microscopy improves the sensitivity of Mtb detection (5). Previously, the light sources necessary for fluorescence microscopy were not available for field use. Recent advances in light emitting diode technology (LED) have widened the applicability of fluorescent microscopy*(6-8). A recent meta-analysis has shown that this technique increases the sensitivity of sputum smears by nearly $10 \%$ over conventional staining (5).

\section{Advances in Culture}

Mycobacterial culture remains the gold standard for detection and drug susceptibility testing. Traditionally, culture on an egg based solid media, known as Lowenstein-Jensen medium, may take as long as 4-6 weeks. Liquid culture systems (BACTEC and MGIT (Becton Dickinson)) offer a more sensitive and rapid alternative to conventional solid culture and may detect growth in 1-3 weeks $*(9,10)$. The mycobacterial growth indicator tube or MGIT contains a fluorescent compound at the bottom of the tube which is quenched by oxygen. As Mtb grows, the oxygen in the tube is used and the fluorescent compound is detected. Potentially, the MGIT technology can yield results in less than 8 days(11). Automated MGIT allows for drug susceptibility through the addition of critical concentrations of streptomycin, INH, rifampin, and ethambutol (12). Currently, the WHO and the Strategic and Technical Advisory Group on TB recommends phased implementation of liquid culture where feasible including low income countries $(4,13)$.

\section{Advances in Nucleic Acid Amplification of Mycobacterium tuberculosis}

Nucleic acid amplification tests (NAAT) offer an important, rapid, and under-utilized molecular tool in Mtb diagnosis. These tests detect mycobacteria with high specificity by amplifying target nucleic acid sequences. The Centers for Disease Control recommends that a NAAT be performed on the first sputum sample of all suspected Mtb patients regardless of smear status(14).

Commercially available NAAT tests include those FDA-approved in smear positive pulmonary diseases (Gen-Probe Amplified Mtb Direct test (GP-AMTD), Roche Amplicor Mtb test (automated version COBAS Amplicor)) and the BD-Probetec Direct (Becton Dickinson). Second generation tests from Gen-Probe (E-AMTD) and Roche (AMTD2) are approved for smear negative specimens. On smear positive samples, the pooled sensitivity is $96 \%$ (15). Sensitivity on smear negative samples has ranged from $66 \%-72(15,16)$. In clinical practice, targeting NAAT technology towards those with a high clinical likelihood of Mtb improved sensitivity estimates with the sensitivity from a multicenter trial reported as 83,75 and $87 \%$ for low, intermediate and high clinical suspicion (17). A recent metaanalysis, looking at over 125 studies, $96 \%$ of which collected smear positive and negative samples, reports a pooled sensitivity and specificity of 85 and $97 \%$ respectively. However, the study noted significant heterogeneity between studies exemplified by the wide variability in sensitivity (36-100\%) and specificity $54-100 \% * *(18)$. 
To obviate the need for reference laboratories, simple and affordable methods have been developed for use in high burden, low technology settings. Loop mediated isothermal amplification (LAMP) from the Eiken group in Japan has been targeted by the Foundation for Innovative New Diagnostics (FIND) as a rapid and deployable NAAT that neither requires thermal cycling nor reagent refrigeration. This isothermal amplification system utilizes multiple primers for increased specificity. Nucleic acid (rRNA) amplification is detected via the release of pyrophosphate byproduct ions which binds with manganese, outcompeting calcein. Calcein, the fluorescent indicator then binds residual magnesium generating stronger fluorescence (19). This approach has been shown to be sensitive in smear positive, culture positive disease (97-100\%) although the sensitivity in smear negative, culture positive disease is reported as $49 \%$. The specificity is reported at $99 \%$ $* *(20,21)$.

\section{Advances in NAATs Detection of Extrapulmonary Tuberculosis}

The use of NAAT in the detection of extrapulmonary TB has been summarized in several meta-analyses $(10,22-24)$. The sensitivity and specificity of NAAT for tuberculous pleural effusions is reported in a meta-analysis at $62 \%$ and $98 \%$ respectively (23) and outperformed the ADA test $(10,25,26)$. The performance of NAAT on cerebrospinal has a pooled a pooled sensitivity and specificity of 56 and $98 \%$ respectively (24).

\section{Advances in the Detection of Drug Resistance}

Perhaps one of the most exciting advances in Mtb diagnostics is rapid drug susceptibility testing (DST). Given the increasing prevalence and impact of multi-drug resistant (MDR) and extensively-drug resistant (XDR) Mtb (27), WHO along with the STOP TB partnership have prioritized greater access to DST. MDR Mtb is defined as resistance to two vital first line agents, rifampin and isoniazid (INH). Rifampin, a rifamycin, inhibits the DNAdependent RNA polymerase (28) and in $96 \%$ of isolates resistant to rifampin, resistance is attributable to a 81 base pair rpo hotspot $(29,30)$. INH is bactericidal against actively replicating mycobacteria by inhibiting pathways of mycolic acid synthesis. It requires activation by the Mtb enzyme katg, a mycobacterial catalase perioxidase, to form reactive intermediates to inhibit various targets of mycolic acid synthesis including InhA, an enoyl acyl carrier protein reductase (31)

Line probe assays (LiPAs) are NAATs that simultaneously detect infection with Mtb and amplify regions of drug resistance. INNO-LiPA®Rif.TB (Innogenetics, Belgium) and GenoType ${ }^{\circledR}$ MTBDR(Hain LifeScience, Germany) are available commercially. LiPAs employ DNA strip technology whereby amplified DNA is applied to strips containing probes specific for Mtb, INH, and rifampin resistance $(32,33)$. WHO has endorsed LiPAs for culture and smear positive clinical specimens as part of a larger commitment to target and implement new technology in high burden regions *(34). The LiPAs are currently not FDA approved.

Although rifampin monoresistance varies among different populations, in regions with a high prevalence of MDR, rifampin tracks with INH resistance in over $90 \%$ of samples (3538). Thus the INNO-LiPA®Rif.TB assay has focused on rifampin resistance as the primary marker for MDRTB. Specifically, the INNO-LiPA®Rif.TB kit simultaneously detects Mtb and the presence of rifampin resistance. Morgan et al (2005) summarized 15 studies on clinical isolates and reported the sensitivity and specificity ranging from $82-100 \%$ and $92-$ $100 \%$ respectively. In contrast, when the kit was used directly with the clinical specimen, the sensitivity ranged from $80-100 \%$ however the specificity was maintained at $100 \%$ (36). 



including (katG) for high level resistance and the promoter region for inhA for low level resistance (33). In a meta-analysis, Ling et al. *(39) reviewed both the first and second generations of the MTBDR assays. The studies included both clinical sputum specimens as well as culture isolates. The pooled sensitivity and specificity was reported as 98.4 and $98 \%$ respectively for rifampin resistance and these estimates did not vary when stratified by studies that looked only at clinical specimens. In contrast, there was more heterogeneity across tests with respect to INH resistance with a pooled sensitivity and specificity of 88.7 and 99.2 respectively. Further, the sensitivity of the MTBDR assays on clinical specimens only in detecting INH resistance decreases to $84 \%$.

Of the studies pooled by Ling et al, the study by Barnard et al. **(40) is notable as it was performed in a highly endemic region, Capetown, South Africa. There was excellent performance of the test in this high burden region with a sensitivity and specificity (RIF/ INH) of 98/94 and 99/99 respectively. In contrast, a recent publication by Lacoma et al. from Spain on the GenoType ${ }^{\circledR}$ MTBDRplus report a sensitivity for INH resistance of $73 \%$ and noting that several false negative isolates found to be resistant fell outside the katG hotspot region (41). However, there is considerable optimism about the LiPA platforms (34) and work is ongoing to ensure that this technology reaches regions with a high Mtb burden (42).

Molecular beacons are an alternate method of rapid detection drug resistance detection. These colorimetric indicators are attractive based on the ease of assay interpretation. Molecular beacons are DNA probes with an intrinsic fluorophore shaped like a hairpin. When bound to the complementary sequence, a conformational change ensues releasing the fluorophore from the quencher, resulting in a detectable visual signal (43). Molecular beacons for Mtb resistance to rifampin and INH from sputum specimens and culture isolates have been reported (44-47) and Lin et al. report a sensitivity and specificity for rifampin resistance of 97 and 100\% while INH resistance was 82 and 100\% respectively on clinical isolates. In addition, there is evidence that they are informative in smear negative, culture positive disease (48). Molecular beacons are currently investigational and not FDA approved. This technology is being further developed by Cepheid Inc. (California) using a kit, Xpert MTB ${ }^{\circledR}$, an automated real time PCR based assay to be performed on sputum samples for Mtb detection and rifampin resistance (49).

The microscopic-observation drug-susceptibility assay (MODS) is a low-cost alternate to the detection of drug resistance. By using Middlebrook 7H9 broth culture containing antituberculous drugs, sputum is directly inoculated and growth (seen as cord formation) is detected using an inverted light microscope. In Ethiopia, MODS detection of MDR TB was excellent with a sensitivity and specificity of 95 and $100 \%$ respectively when compared with the MGIT 960 system *(50). The time to detection has been shown to be 7 days (51) and similar to the MGIT $960 *(52)$. A large study in Peru reports excellent sensitivity of the MODS at $97.8 \%$, nearly $10 \%$ greater than automated mycobacterial culture and LJ media (51). When used for DST, the median time for susceptibility testing for MODS mirrored time to detection (7 days) followed by DST at 22 days for automated culture.

\section{Advances in the Detection of Latent TB}

It is estimated that at least one half of all tuberculosis cases are the result of reactivation of latent TB. As a result, the detection and treatment of LTBI remains a public health priority $(53,54)$. Here, testing for LTBI is performed with the intention to treat those who test positive, and consequently is focused on those likely to be infected with Mtb (contacts of active cases and those from endemic area) with particular attention to those at high risk for 
disease progression (recent evidence of exposure, young age, and immunocompromised). At present, targeted testing for LTBI is recommended in settings where the prevalence of tuberculosis is relatively low and comments in this section will be focused on the use of IGRAs in these settings.

Until recently, the detection of LTBI has relied upon skin test reactivity to a mixture of antigens derived from Mtb (PPD). While TST testing has proven useful in the detection and treatment of LTBI, difficulties regarding the placement, reading, recording, and interpretation of the TST are well known. In this regard, it is sobering to note that treatment of LTBI based upon TST testing is completed in less than $1 / 3$ of those cases where targeted testing is recommended $(55,56)$. One issue that has persistently dogged the TST is the perception of its diminished specificity, particularly in those given the BCG vaccine. In this regard, blood tests based upon immune responses to the specific Mtb antigens ESAT- 6 and CFP-10, absent from most environmental mycobacteria and BCG, are of particular interest. These assays rely on the detection of interferon gamma released by sensitized $\mathrm{T}$ cells (largely CD4 T cells) in response to these antigens and are collectively called interferon gamma release assays (IGRAs). Currently there are two commercially available FDAapproved platforms, the Quantiferon In-Tube ${ }^{\circledR}$ (Cellestis Australia) and the T.SPOT.TB ${ }^{\circledR}$ (Oxford Immunotech). The Quantiferon-Gold (QFT-G; $2^{\text {nd }}$ generation) and the current Quantiferon In-Tube ${ }^{\circledR}$ assay (QFT-IT) is a whole blood format that uses an ELISA to detect interferon-gamma while the T.SPOT.TB ${ }^{\circledR}$ uses purified peripheral blood mononuclear cells (PBMC) with an enzyme-linked immunospot (ELISPOT) technique.

\section{IGRAs: Test Performance and Characteristics}

In clinical practice, IGRAs have been studied in both LTBI and active disease in different geographic settings. In interpreting test performance, it is useful to look at test performance in geographic regions that test for LTBI, historically low burden regions.

Like the TST, the quantity of IFN- $\gamma$ released in is a quantitative reflection of the T cell response to Mtb-derived antigens. Historically, differing TST thresholds have been employed to help the clinician balance the pre-test likelihood of true infection with Mtb versus antigenic cross-reactivity with BCG and/or environmental mycobacteria. In addition to threshold values that define a positive IGRA, IGRAs include both media (nil) as well as mitogen controls to aid in the interpretation of these tests. Unlike the TST, the IGRAs are reported as positive, negative or indeterminate. The indeterminate category can reflect a high media response or poor response to mitogen (anergy), adding an important category especially in immune compromised hosts. FDA-approved cut offs for a positive test have been validated for both the Quantiferon and T.SPOT assays $(57,58)$. In this regard, it is important to note that the cutoffs for each test have been differently calibrated. The $0.35 \mathrm{IU}$ cutoff for the QFT-IT allows for high specificity, but likely some cost in sensitivity (59). Conversely, the current FDA cutoffs for T-SPOT.TB are $\leq 4$ spots - negative; 5-7 spots "equivocal or borderline" and $\geq 8$ spots positive has been calibrated to allow for greater sensitivity (60). Published data for the T-SPOT.TB have used the six spot cut off.

A recent meta-analysis by Pai et al reports the pooled specificity of the QFT-G as $99 \%$, the QFT-IT as $96 \%$ and the T.SPOT as $93 \% * *(61)$. For the QFTs the sensitivity of the QFT-G and QFT-IT have recently been reported as 81.4 and $92.6 \%$ respectively (59) although in pooled sensitivity estimates from multiple studies in different geographic regions, the estimates have been lower (as 78 and $70 \%$ respectively) (61). For the T-SPOT.TB test, Pai et al report the pooled sensitivity of $90 \%$ (61). The recent FDA approval of the T.SPOT is notable for a change in the threshold for a positive test (as discussed above) and the current T.SPOT package insert reports a sensitivity and specificity of 95 and $96 \%$ respectively (57). 
Head to head studies with the T.SPOT.TB and QFT-G have suggested somewhat better T.SPOT.TB sensitivity (62-64). As with the TST, a negative IGRA does not rule out Mtb disease, and should be regarded as an adjunct not a substitute for the clinical evaluation of those suspected of having tuberculosis.

Because the IGRAs (like the TST) reflect the host response to underlying infection, there is likely to be both underlying biologic variation in the immune response as well as inherent variability in the determination of IFN- $\gamma$. For example, a variance of $15 \%$ is considered acceptable by the FDA. For these reasons it is important that the clinician receive quantitative information from the assay so as to allow for appropriate interpretation. For example in subjects classified as low risk for infection with Mtb, values at or near the cutoff values should be interpreted cautiously. Similarly, "conversions" that occur near these thresholds should be interpreted with caution. At present, there are insufficient data to allow for quantitative guidance on these issues.

\section{Clinical Use of IGRAs}

While there remains no gold standard for LTBI, when surrogate markers of recent exposure to Mtb such as proximity to an active case are used in low burden regions, IGRAs have been shown in multiple studies to be as good if not better than the TST in a contact investigation *(65-71). These data in conjunction with the performance characteristics of IGRAs led the CDC to recommend that these assays may be used in all situations that the TST is used, but cautioned against its use in the setting of severe immunocompromise and the very young (72). Clearly, data regarding risk of disease progression is incomplete, but recent data would suggest that a positive IGRA may predict future progression ** $(73,74)$. Given the diagnostic accuracy of IGRAs in the setting on active TB, their improved association with surrogates of Mtb exposure, the low likelihood of completion of INH therapy based in TST testing, and the absence of data suggesting that IGRA testing misses those at risk for disease progression, it is the opinion of the authors that the use of IGRAs, where available, is preferred to TST testing in all situations currently recommended for targeted testing by the CDC. This recommendation is further based on the potential for harm inherent in the administration of INH in those with false positive TST tests. This would include persons from Mtb-endemic regions, particularly where BCG vaccination is employed, those potentially recently exposed to $\mathrm{Mtb}$, and those with radiographic evidence of prior infection with Mtb. Specific limitations and cautions are discussed below.

\section{Immunocompromised persons}

Immunocompromised persons are at high risk to reactivate latent disease with an estimated risk of $10 \%$ per year. Given this, the CDC currently recommends targeted testing for LTBI in specific at risk groups including individuals with HIV/AIDS, chronic renal failure, solid organ transplant, fibrotic lesions, silicosis, cancer of the head and neck, malnutrition and diabetes (53). While the TST is well known to have diminished sensitivity in the setting of immunocompromised hosts, limited data are available for the use of IGRAs in this setting. For the QFT-Gold, Kobayashi et al. evaluated a heterogeneous group of immunocompromised patients and obtained a sensitivity for the detection of tuberculosis of 25/32 (78\%) compared to 16/32 for TST (50\%) (75). For T-SPOT.TB, Clark et al reported a sensitivity of $90.3 \%$ in HIV patients with CD4 T cell counts < 300, half of which were < 200 (76), and in one study that employed both QFT-Gold and T-SPOT.TB had identical sensitivities of $84.6 \%$ (11/13) (77). These limited data would suggest that IGRAs are at least as sensitive as TST in the immunocompromised setting.

Studies that have compared IGRAs to TST in routine testing for LTBI are very heterogeneous with regard to the type of underlying immonocompromise and the reasons for 
testing. Nonetheless, these studies demonstrate significant discord between TST and IGRA results. There are little data using surrogate markers of exposure to help elucidate the source of this discord. In the setting of advanced HIV infection the T.SPOT.TB appears the more resilient assay (78-82) although may be limited with CD4 T cells that are $<50$ (83).

Several conclusions may be drawn on the use of IGRAs in immunocompromised persons. As in immune competent persons, IGRAs are not a substitute for clinical judgment. Second, to maximize sensitivity in an immune compromised individual with high risk of Mtb infection, it is reasonable to perform both an IGRA and the TST. In this setting, the clinician would be advised to accept a positive result from either test as evidence for infection with Mtb. We do not recommend stepwise testing (ie TST followed by an IGRA) as this may miss an important group of IGRA+/TST- persons.

One area of uncertainty is in the management of subjects prior to the initiation of potent immunosuppressive therapy. This might include those being considered for anti-TNF-a therapy (84) or those being considered for liquid or solid organ transplantation. While the greatest risk of TB disease is traditionally associated with recent exposures and/or infection, this is a setting in which reactivation of TB from more temporally remote exposures is a concern. While testing for LTBI in this setting is recommended, there is insufficient information to make a firm recommendation. In one recent report, Bocchini et al. recently reported good sensitivity of the QFT and T-SPOT in rheumatologic patients prior to antiTNF therapy, where in this cohort, the T.SPOT had a slightly higher proportion of indeterminate results versus the QFT-IT (5.8\% vs. $2.8 \%$ respectively)(85).

\section{Those thought to be at low risk of infection and/or progression}

At present, CDC guidelines do not recommend testing in this group. However, the TST is currently performed in many groups thought to be at low risk (employee and school screening). The excellent specificity of IGRAs would favor their use in this setting. However, where longitudinal testing is performed, there is very little information to guide interpretation of the test in this setting. In geographic areas where Mtb is endemic, "conversions" and "reversions" have been documented and often occur near the threshold values of the test. The prevalence of tests occurring near these values in areas of low Mtb burden, and the likelihood of test conversions and reversions is currently not known, such that caution in interpretation of values at or near the threshold is advised. Thus, because of the low likelihood of a false positive with an IGRA, performing a blood test in place of a TST may obviate the need for further clinical testing (x-rays) and INH. However, given the data on conversions/or reversions as well as the variability allowed by the FDA (15\%), a positive test in this low risk setting should be considered carefully with review of the quantitative IGRA result and weighed against the likelihood of new infection.

\section{Children}

School age children have a similar risk of developing TB as adults and display a similar disease spectrum (86). In addition, older children react similarly to adults to the TST. Therefore, much of what has been learned in adults regarding IGRA performance can be extrapolated to older children. However, children $<5$ years are at increased risk of developing TB following infection and more likely to develop severe disease than older children and adults and so must be studied separately. In this regard the numbers of studies of IGRA performance in children are growing, albeit still limited. In general, the results are consistent with results of studies of IGRA performance in adults in that the sensitivity of IGRAs in children with TB (87-91) and in older children who are household $(92,93)$ or school contacts(94), and the specificity of IGRAs in children $(89,94)$ are comparable to adults. Moreover, IGRAs may be helpful in clinical scenarios where TST performs 
especially poorly, such as congenital TB (95) or in very young children with HIV infection and/or malnutrition (81). However, the number of young children studied remains limited, and even fewer children from non-endemic regions have been studied. Moreover, indeterminate results may be more frequently observed in young children $(63,87,96)$ and test failure due to inadequate phlebotomy is more common in young children $(92,97)$. Hence, IGRAs can be used as they are in adults in school age children. However, IGRAs should still be used with caution in younger children due to limitations in our knowledge of test performance in this age group.

Children under age 5 are at high risk of disease progression following exposure, and there is currently very little information to support their routine use, particularly in the setting of household exposure. Children over age five are considered immunologically mature, and recommendations for children are parallel to those offered for adults above.

\section{Biomarkers}

While this review has highlighted improvements in the detection of drug sensitive and resistant Mtb, and in the accurate identification of those with LTBI, tests capable of rapidly and inexpensively identifying those with Mtb disease, delineating appropriate responses to drug therapy, and those at risk for disease progression are desperately needed. Generically, these have been termed biomarkers, and ideally could function as a point of care test. Much work has been done on serodiagnostics using Mtb specific antibodies to detect disease states. Areas of active investigation include: New antibodies of potential use as a serodiagnostic (98)(99) (100), (101); the CXC chemokine IP-10 (interferon gamma inducible protein) *(102); the novel T cell antigen Mtb heparin binding heme agglutinin (HBHA) that has been suggested to be LTBI-associated (103) ; and renewed interest in skin testing using the Mtb-seletive antigen ESAT-6 (104); and the use of novel T cell subsets and cytokine profiles; as well as the detection of Mtb products from urine (105).

\section{Conclusions}

Improvement in the identification of the spectrum of Mtb infection, including active, latent and drug resistant strains, has the potential to have a profound effect on global health. For example, a test with $85 \%$ sensitivity and $97 \%$ specificity in the identification of those with TB could prevent 392,000 deaths annually ( $22 \%$ of global TB deaths) (106). Continued research into Mtb diagnostics is needed with a view towards sensitive, specific, and easily deployable assays.

\section{Acknowledgments}

Funding: MRN: NIH/NCRR 5 KL2 RR024141-03

DAL: NIH HHSN266200400081C (N01-A1-400081), RO1AI054474

DML: VA Merit Review Grant, Portland VA Medical Center, NIH HHSN266200400081C (N01-A1-400081), RO1AI48090

\section{References}

Studies referenced in 2007-2008

*Special interest

** Outstanding interest 
1. WHO. Global tuberculosis control: surveillance, planning, financing: WHO report. 2008. WHO/ HTM/TB/20083932008

2. Dye C. Global epidemiology of tuberculosis. Lancet. Mar 18; 2006 367(9514):938-40. [PubMed: 16546542]

3. Iseman MD. TB elimination in the 21st century, a quixotic dream? Int J Tuberc Lung Dis. Dec; 2000 4(12 Suppl 2):S109-10. [PubMed: 11144538]

4. WHO. Strategic and Technical Advisory Group For Tuberculosis Geneva. 2007. Report on Conclusions and Recommendations.

5. Steingart KR, Henry M, Ng V, Hopewell PC, Ramsay A, Cunningham J, et al. Fluorescence versus conventional sputum smear microscopy for tuberculosis: a systematic review. Lancet Infect Dis. Sep; 2006 6(9):570-81. [PubMed: 16931408]

6. Anthony RM, Kolk AH, Kuijper S, Klatser PR. Light emitting diodes for auramine O fluorescence microscopic screening of Mycobacterium tuberculosis. Int J Tuberc Lung Dis. Sep; 2006 10(9): 1060-2. [PubMed: 16964802]

7. Marais BJ, Brittle W, Painczyk K, Hesseling AC, Beyers N, Wasserman E, et al. Use of lightemitting diode fluorescence microscopy to detect acid-fast bacilli in sputum. Clinical Infectious Diseases. Jul 15; 2008 47(2):203-7. [PubMed: 18532893] *Reports on the sensitivity and specificity of LED compared with MVP

8. Van Deun A, Chonde TM, Gumusboga M, Rienthong S. Performance and acceptability of the FluoLED Easy module for tuberculosis fluorescence microscopy. Int J Tuberc Lung Dis. Sep; 2008 12(9):1009-14. [PubMed: 18713497]

9. Cruciani M, Scarparo C, Malena M, Bosco O, Serpelloni G, Mengoli C. Meta-analysis of BACTEC MGIT 960 and BACTEC 460 TB, with or without solid media, for detection of mycobacteria. J Clin Microbiol. May; 2004 42(5):2321-5. [PubMed: 15131224]

10. Dinnes J, Deeks J, Kunst H, Gibson A, Cummins E, Waugh N, et al. A systematic review of rapid diagnostic tests for the detection of tuberculosis infection. Health Technol Assess. Jan; 2007 11(3): 1-196. [PubMed: 17266837] *Large reference for many rapid diagnostic tests, sensitivity and specificity.

11. Palomino JC, Martin A, Von Groll A, Portaels F. Rapid culture-based methods for drug-resistance detection in Mycobacterium tuberculosis. J Microbiol Methods. Oct; 2008 75(2):161-6. [PubMed: 18627779]

12. Becton Dickinson - Package Insert BACTEC MGIT 960 SIRE KIT. 2007. Anonymous

13. Siddiqi, R-G. Foundation for Innovative New Diagnostics. 2006. MGIT Procedure Manual.

14. Notice to Readers: Update: Nucelic Acid Amplification Tests for Tuberculosis. MMWR. 2000; 49(26):593-4. Anonymous. [PubMed: 10921499]

15. Greco S, Girardi E, Navarra A, Saltini C. Current evidence on diagnostic accuracy of commercially based nucleic acid amplification tests for the diagnosis of pulmonary tuberculosis. Thorax. Sep; 2006 61(9):783-90. [PubMed: 16738037]

16. Sarmiento OL, Weigle KA, Alexander J, Weber DJ, Miller WC. Assessment by meta-analysis of PCR for diagnosis of smear-negative pulmonary tuberculosis. J Clin Microbiol. Jul; 2003 41(7): 3233-40. [PubMed: 12843069]

17. Catanzaro A, Perry S, Clarridge JE, Dunbar S, Goodnight-White S, LoBue PA, et al. The role of clinical suspicion in evaluating a new diagnostic test for active tuberculosis: results of a multicenter prospective trial. JAMA. Feb 2; 2000 283(5):639-45. [PubMed: 10665704]

18. Ling DI, Flores LL, Riley LW, Pai M, Ling DI, Flores LL, et al. Commercial nucleic-acid amplification tests for diagnosis of pulmonary tuberculosis in respiratory specimens: meta-analysis and meta-regression. PLoS ONE. 2008; 3(2):e1536. [PubMed: 18253484] *Meta-analysis on the performance of the commercial NAAT also cited by the STOP-TB partnership in a letter detailing the importance of synthesis of high quality diagnostic studies.

19. Tomita N, Mori Y, Kanda H, Notomi T. Loop-mediated isothermal amplification (LAMP) of gene sequences and simple visual detection of products. Nat Protoc. 2008; 3(5):877-82. [PubMed: 18451795]

20. Boehme CC, Nabeta P, Henostroza G, Raqib R, Rahim Z, Gerhardt M, et al. Operational feasibility of using loop-mediated isothermal amplification for diagnosis of pulmonary tuberculosis in 
microscopy centers of developing countries. J Clin Microbiol. Jun; 2007 45(6):1936-40. [PubMed: 17392443] *Operational applicability of the new technology in multiple sites including Peru, Bangladesh, and Tanzania

21. Pandey BD, Poudel A, Yoda T, Tamaru A, Oda N, Fukushima Y, et al. Development of an inhouse loop-mediated isothermal amplification (LAMP) assay for detection of Mycobacterium tuberculosis and evaluation in sputum samples of Nepalese patients. J Med Microbiol. Apr; 2008 57(Pt 4):439-43. [PubMed: 18349362] *Sensitivity and Specificity estimates of LAMP technology in a high burden region.

22. Daley P, Thomas S, Pai M. Nucleic acid amplification tests for the diagnosis of tuberculous lymphadenitis: a systematic review. International Journal of Tuberculosis \& Lung Disease. Nov; 2007 11(11):1166-76. Review. [PubMed: 17958977]

23. Pai M, Flores LL, Hubbard A, Riley LW, Colford JM Jr. Nucleic acid amplification tests in the diagnosis of tuberculous pleuritis: a systematic review and meta-analysis. BMC Infect Dis. Feb. 2004 23(4):6. [PubMed: 15102325]

24. Pai M, Flores LL, Pai N, Hubbard A, Riley LW, Colford JM Jr. Diagnostic accuracy of nucleic acid amplification tests for tuberculous meningitis: a systematic review and meta-analysis. Lancet Infect Dis. Oct; 2003 3(10):633-43. [PubMed: 14522262]

25. Nagesh BS, Sehgal S, Jindal SK, Arora SK. Evaluation of polymerase chain reaction for detection of Mycobacterium tuberculosis in pleural fluid. Chest. Jun; 2001 119(6):1737-41. [PubMed: 11399699]

26. Villegas MV, Labrada LA, Saravia NG. Evaluation of polymerase chain reaction, adenosine deaminase, and interferon-gamma in pleural fluid for the differential diagnosis of pleural tuberculosis. Chest. Nov; 2000 118(5):1355-64. [PubMed: 11083686]

27. Dorman SE, Chaisson RE. From magic bullets back to the magic mountain: the rise of extensively drug-resistant tuberculosis. Nat Med. Mar; 2007 13(3):295-8. [PubMed: 17342143]

28. Mandell, B.; Dolin, editors. Principles and Practice of Infectious Diseases. 6 ed. Elsevier; Philidelphia: 2005.

29. Heep M, Brandstatter B, Rieger U, Lehn N, Richter E, Rusch-Gerdes S, et al. Frequency of rpoB mutations inside and outside the cluster I region in rifampin-resistant clinical Mycobacterium tuberculosis isolates. J Clin Microbiol. Jan; 2001 39(1):107-10. [PubMed: 11136757]

30. Moghazeh SL, Pan X, Arain T, Stover CK, Musser JM, Kreiswirth BN. Comparative antimycobacterial activities of rifampin, rifapentine, and KRM-1648 against a collection of rifampin-resistant Mycobacterium tuberculosis isolates with known rpoB mutations. Antimicrob Agents Chemother. Nov; 1996 40(11):2655-7. [PubMed: 8913484]

31. Timmins GS, Deretic V. Mechanisms of action of isoniazid. Mol Microbiol. Dec; 2006 62(5): 1220-7. [PubMed: 17074073]

32. 2008. Anonymous. INNO-LiPA Rif.TB: http://www.innogenetics.com/infectiousdiseases.html? id=22cited 2008

33. 2008. Anonymous. Hain-Lifescience Genotype MTBDR-plus Package Insert http://www.hainlifescience.com/pdf/304xx_pbl.pdf

34. WHO. 2008Molecular Line Probe Assays For Rapid Screening of Patients at Risk of MDR-TB: http://www.who.int/tb/features_archive/policy_statement.pdf *Important statement by WHO on the future deployment of LiPAs.

35. Jiao WW, Mokrousov I, Sun GZ, Li M, Liu JW, Narvskaya O, et al. Molecular characteristics of rifampin and isoniazid resistant Mycobacterium tuberculosis strains from Beijing, China. Chin Med J (Engl). May 5; 2007 120(9):814-9. [PubMed: 17531124]

36. Morgan M, Kalantri S, Flores L, Pai M. A commercial line probe assay for the rapid detection of rifampicin resistance in Mycobacterium tuberculosis: a systematic review and meta-analysis. BMC Infect Dis. 2005; 5:62. [PubMed: 16050959]

37. Traore H, Fissette K, Bastian I, Devleeschouwer M, Portaels F. Detection of rifampicin resistance in Mycobacterium tuberculosis isolates from diverse countries by a commercial line probe assay as an initial indicator of multidrug resistance. Int J Tuberc Lung Dis. May; 2000 4(5):481-4. [PubMed: 10815743] 
38. Wright, AF. 2007. Does rifampicin resistance reliably predict MDR-TB? Results from the Global Project: http://www.finddiagnostics.org/news/presentations/iuatld_riga_conf2007/ R_MDR_a_wright.pdf

39. Ling DI, Zwerling AA, Pai M. GenoType MTBDR assays for the diagnosis of multidrug-resistant tuberculosis: a meta-analysis. Eur Respir J. Nov; 2008 32(5):1165-74. [PubMed: 18614561]

*Meta-analysis for sensitivity /specificity of commercially available Line Probe Assays.

40. Barnard M, Albert H, Coetzee G, O'Brien R, Bosman ME. Rapid molecular screening for multidrug-resistant tuberculosis in a high-volume public health laboratory in South Africa. Am J Respir Crit Care Med. Apr 1; 2008 177(7):787-92. [PubMed: 18202343] **Performance of LiPAs in high volume, high burden setting.

41. Lacoma A, Garcia-Sierra N, Prat C, Ruiz-Manzano J, Haba L, Roses S, et al. GenoType MTBDRplus assay for molecular detection of rifampin and isoniazid resistance in Mycobacterium tuberculosis strains and clinical samples. J Clin Microbiol. Nov; 2008 46(11):3660-7. [PubMed: 18784319]

42. WHO. New Techonologies for Tuberculosis control: a framework for their adoption, introduction and implementation. WHO/HTM/STB/2007.402007

43. Tan W, Wang K, Drake TJ, Tan W, Wang K, Drake TJ. Molecular beacons. Current Opinion in Chemical Biology. Oct; 2004 8(5):547-53. [PubMed: 15450499]

44. El-Hajj HH, Marras SA, Tyagi S, Kramer FR, Alland D. Detection of rifampin resistance in Mycobacterium tuberculosis in a single tube with molecular beacons. J Clin Microbiol. Nov; 2001 39(11):4131-7. [PubMed: 11682541]

45. Lin SY, Probert W, Lo M, Desmond E. Rapid detection of isoniazid and rifampin resistance mutations in Mycobacterium tuberculosis complex from cultures or smear-positive sputa by use of molecular beacons. J Clin Microbiol. Sep; 2004 42(9):4204-8. [PubMed: 15365012]

46. Piatek AS, Telenti A, Murray MR, El-Hajj H, Jacobs WR Jr, Kramer FR, et al. Genotypic analysis of Mycobacterium tuberculosis in two distinct populations using molecular beacons: implications for rapid susceptibility testing. Antimicrob Agents Chemother. Jan; 2000 44(1):103-10. [PubMed: 10602730]

47. Varma-Basil M, El-Hajj H, Marras SA, Hazbon MH, Mann JM, Connell ND, et al. Molecular beacons for multiplex detection of four bacterial bioterrorism agents. Clin Chem. Jun; 2004 50(6): 1060-2. [PubMed: 15161722]

48. Haldar S, Chakravorty S, Bhalla M, De Majumdar S, Tyagi JS, Haldar S, et al. Simplified detection of Mycobacterium tuberculosis in sputum using smear microscopy and PCR with molecular beacons. Journal of Medical Microbiology. Oct; 2007 56(Pt 10):1356-62. [PubMed: 17893174]

49. [(accessed November, 2008)] 2008. Anonymous. FIND: http://www.finddiagnostics.org/activities/ tb/tb_pipeline.shtml

50. Ejigu GS, Woldeamanuel Y, Shah NS, Gebyehu M, Selassie A, Lemma E. Microscopicobservation drug susceptibility assay provides rapid and reliable identification of MDR-TB. Int J Tuberc Lung Dis. Mar; 2008 12(3):332-7. [PubMed: 18284841]

51. Moore DA, Evans CA, Gilman RH, Caviedes L, Coronel J, Vivar A, et al. Microscopicobservation drug-susceptibility assay for the diagnosis of TB. N Engl J Med. Oct 12; 2006 355(15):1539-50. [PubMed: 17035648]

52. Arias M, Mello FC, Pavon A, Marsico AG, Alvarado-Galvez C, Rosales S, et al. Clinical evaluation of the microscopic-observation drug-susceptibility assay for detection of tuberculosis. Clin Infect Dis. Mar 1; 2007 44(5):674-80. [PubMed: 17278058]

53. CDC. Targeted Tuberculin Testing and Treatment of Latent Tuberculosis Infection. MMWR. 2000; 49(RR06):1-54.

54. Geiter, L., editor. Ending Neglect: The Elimination of Tuberculosis from the United States. National Academy Press; Washington, D.C.: 2000.

55. Marks SM, Taylor Z, Qualls NL, Shrestha-Kuwahara RJ, Wilce MA, Nguyen CH. Outcomes of contact investigations of infectious tuberculosis patients. Am J Respir Crit Care Med. Dec; 2000 162(6):2033-8. [PubMed: 11112109] 
56. Reichler MR, Reves R, Bur S, Ford J, Thompson V, Mangura B, et al. Treatment of latent tuberculosis infection in contacts of new tuberculosis cases in the United States. South Med J. Apr; 2002 95(4):414-20. [PubMed: 11958239]

57. Oxford, UK: 2008. Anonymous. T-SPOT.TB PACKAGE INSERT http:// www.oxfordimmunotec.com/eu/downloads/PI-TB96-IVD-UK-V8,\%20English.pdf

58. Nov. 2008 Anonymous. Quantiferon In-Tube Package Insert : http://www.cellestis.com/IRM/ Company/ShowPage.aspx?CPID=1171. 2008

59. Harada N, Higuchi K, Yoshiyama T, Kawabe Y, Fujita A, Sasaki Y, et al. Comparison of the sensitivity and specificity of two whole blood interferon-gamma assays for M. tuberculosis infection. J Infect. May; 2008 56(5):348-53. [PubMed: 18395264]

60. FDA. 2008. Summary of Safety and Effectiveness: T-SPOT.TB http://www.fda.gov/cdrh/pdf7/ p070006b.pdfcited 2008

61. Pai M, Zwerling A, Menzies D, Pai M, Zwerling A, Menzies D. Systematic review: T-cell-based assays for the diagnosis of latent tuberculosis infection: an update. Annals of Internal Medicine. Aug 5; 2008 149(3):177-84. [PubMed: 18593687] **Recent meta-analysis of cumulative data for sensitivity and specificity analysis for commercial IGRAs.

62. Chee CB, Gan SH, Khinmar KW, Barkham TM, Koh CK, Liang S, et al. Comparison of sensitivities of two commercial gamma interferon release assays for pulmonary tuberculosis. $\mathrm{J}$ Clin Microbiol. Jun; 2008 46(6):1935-40. [PubMed: 18400912]

63. Ferrara G, Losi M, D'Amico R, Roversi P, Piro R, Meacci M, et al. Use in routine clinical practice of two commercial blood tests for diagnosis of infection with Mycobacterium tuberculosis: a prospective study. Lancet. Apr 22; 2006 367(9519):1328-34. [PubMed: 16631911]

64. Kobashi Y, Mouri K, Yagi S, Obase Y, Miyashita N, Okimoto N, et al. Clinical evaluation for diagnosing active TB disease and transitional change of two commercial blood tests. Scand $\mathrm{J}$ Infect Dis. 2008; 40(8):629-34. [PubMed: 18642159]

65. Arend SM, Thijsen SF, Leyten EM, Bouwman JJ, Franken WP, Koster BF, et al. Comparison of two interferon-gamma assays and tuberculin skin test for tracing tuberculosis contacts. Am J Respir Crit Care Med. Mar 15; 2007 175(6):618-27. [PubMed: 17170386] *Large contact evaluation in low burden country reviewing performance of both commercially available IGRAs.

66. Diel R, Ernst M, Doscher G, Visuri-Karbe L, Greinert U, Niemann S, et al. Avoiding the effect of BCG-vaccination in detecting MTB infection with a blood test. Eur Respir J. Feb 15.2006

67. Ewer K, Deeks J, Alvarez L, Bryant G, Waller S, Andersen P, et al. Comparison of T-cell-based assay with tuberculin skin test for diagnosis of Mycobacterium tuberculosis infection in a school tuberculosis outbreak. Lancet. Apr 5; 2003 361(9364):1168-73. [PubMed: 12686038]

68. Harada N, Nakajima Y, Higuchi K, Sekiya Y, Rothel J, Mori T. Screening for tuberculosis infection using whole-blood interferon-gamma and Mantoux testing among Japanese healthcare workers. Infect Control Hosp Epidemiol. May; 2006 27(5):442-8. [PubMed: 16671023]

69. Lalvani A, Pathan AA, Durkan H, Wilkinson KA, Whelan A, Deeks JJ, et al. Enhanced contact tracing and spatial tracking of Mycobacterium tuberculosis infection by enumeration of antigenspecific T cells. Lancet. Jun 23; 2001 357(9273):2017-21. [PubMed: 11438135]

70. Shams H, Weis SE, Klucar P, Lalvani A, Moonan PK, Pogoda JM, et al. Enzyme-Linked Immunospot and Tuberculin Skin Testing to Detect Latent Tuberculosis Infection. Am J Respir Crit Care Med. Aug 4.2005

71. Diel R, Loddenkemper R, Meywald-Walter K, Gottschalk R, Nienhaus A. Comparative Performance of Tuberculin Skin Test, QuantiFERON-TB-Gold In Tube Assay, and T-Spot.TB Test in Contact Investigations for Tuberculosis. Chest. Nov 18.2008

72. Mazurek GH, Jereb J, Lobue P, Iademarco MF, Metchock B, Vernon A. Guidelines for using the QuantiFERON-TB Gold test for detecting Mycobacterium tuberculosis infection, United States. MMWR Recomm Rep. Dec 16; 2005 54(RR-15):49-55. [PubMed: 16357824]

73. Diel R, Loddenkemper R, Meywald-Walter K, Niemann S, Nienhaus A. Predictive value of a whole blood IFN-gamma assay for the development of active tuberculosis disease after recent infection with Mycobacterium tuberculosis. Am J Respir Crit Care Med. May 15; 2008 177(10): 1164-70. [PubMed: 18276940] *The natural history of a postive IGRA is not currently known, but new data, such as this publication suggests that a positive IGRA may predict future active disease. 
74. Doherty TM, Demissie A, Olobo J, Wolday D, Britton S, Eguale T, et al. Immune responses to the Mycobacterium tuberculosis-specific antigen ESAT-6 signal subclinical infection among contacts of tuberculosis patients. J Clin Microbiol. Feb; 2002 40(2):704-6. [PubMed: 11826002]

75. Kobashi Y, Mouri K, Obase Y, Fukuda M, Miyashita N, Oka M. Clinical evaluation of QuantiFERON TB-2G test for immunocompromised patients. Eur Respir J. Nov; 2007 30(5):94550. [PubMed: 17652312]

76. Clark SA, Martin SL, Pozniak A, Steel A, Ward B, Dunning J, et al. Tuberculosis antigen-specific immune responses can be detected using enzyme-linked immunospot technology in human immunodeficiency virus (HIV)-1 patients with advanced disease. Clin Exp Immunol. Nov; 2007 150(2):238-44. [PubMed: 17672869]

77. Vincenti D, Carrara S, Butera O, Bizzoni F, Casetti R, Girardi E, et al. Response to region of difference 1 (RD1) epitopes in human immunodeficiency virus (HIV)-infected individuals enrolled with suspected active tuberculosis: a pilot study. Clin Exp Immunol. Oct; 2007 150(1):91-8. [PubMed: 17680823]

78. Chapman AL, Munkanta M, Wilkinson KA, Pathan AA, Ewer K, Ayles H, et al. Rapid detection of active and latent tuberculosis infection in HIV-positive individuals by enumeration of Mycobacterium tuberculosis-specific T cells. AIDS. Nov 22; 2002 16(17):2285-93. [PubMed: 12441800]

79. Dheda K, Lalvani A, Miller RF, Scott G, Booth H, Johnson MA, et al. Performance of a T-cellbased diagnostic test for tuberculosis infection in HIV-infected individuals is independent of CD4 cell count. AIDS. Nov 18; 2005 19(17):2038-41. [PubMed: 16260914]

80. Lawn SD, Bangani N, Vogt M, Bekker LG, Badri M, Ntobongwana M, et al. Utility of interferongamma ELISPOT assay responses in highly tuberculosis-exposed patients with advanced HIV infection in South Africa. BMC Infect Dis. 2007; 7:99. [PubMed: 17725839]

81. Liebeschuetz S, Bamber S, Ewer K, Deeks J, Pathan AA, Lalvani A. Diagnosis of tuberculosis in South African children with a T-cell-based assay: a prospective cohort study. Lancet. Dec 18-31; 2004 364(9452):2196-203. [PubMed: 15610806]

82. Hammond AS, McConkey SJ, Hill PC, Crozier S, Klein MR, Adegbola RA, et al. Mycobacterial T cell responses in HIV-infected patients with advanced immunosuppression. J Infect Dis. Jan 15; 2008 197(2):295-9. [PubMed: 18194089]

83. Karam F, Mbow F, Fletcher H, Senghor CS, Coulibaly KD, LeFevre AM, et al. Sensitivity of IFNgamma release assay to detect latent tuberculosis infection is retained in HIV-infected patients but dependent on HIV/AIDS progression. PLoS ONE. 2008; 3(1):e1441. [PubMed: 18197251]

84. Keane J. TNF-blocking agents and tuberculosis: new drugs illuminate an old topic. Rheumatology (Oxford). Jun; 2005 44(6):714-20. [PubMed: 15741198]

85. Bocchino M, Matarese A, Bellofiore B, Giacomelli P, Santoro G, Balato N, et al. Performance of two commercial blood IFN-gamma release assays for the detection of Mycobacterium tuberculosis infection in patient candidates for anti-TNF-alpha treatment. Eur J Clin Microbiol Infect Dis. Oct; 2008 27(10):907-13. [PubMed: 18470544] *The use of IGRAs to diagnose LTBI prior to immunosuppressive treatment is novel and this study shows that the IGRAs picked up persons with TB risk factors that the TST did not.

86. Comstock GW, Livesay VT, Woolpert SF. The prognosis of a positive tuberculin reaction in childhood and adolescence. Am J Epidemiol. Feb; 1974 99(2):131-8. [PubMed: 4810628]

87. Connell TG, Curtis N, Ranganathan SC, Buttery JP. Performance of a whole blood interferon gamma assay for detecting latent infection with Mycobacterium tuberculosis in children. Thorax. Jul; 2006 61(7):616-20. [PubMed: 16601088]

88. Connell TG, Ritz N, Paxton GA, Buttery JP, Curtis N, Ranganathan SC. A three-way comparison of tuberculin skin testing, QuantiFERON-TB gold and T-SPOT.TB in children. PLoS ONE. 2008; 3(7):e2624. [PubMed: 18612425]

89. Detjen AK, Keil T, Roll S, Hauer B, Mauch H, Wahn U, et al. Interferon-gamma release assays improve the diagnosis of tuberculosis and nontuberculous mycobacterial disease in children in a country with a low incidence of tuberculosis. Clin Infect Dis. Aug 1; 2007 45(3):322-8. [PubMed: 17599309] **Provides important specificity data of the IGRAs in a pediatric cohort in a low incidence country. 
90. Dogra S, Narang P, Mendiratta DK, Chaturvedi P, Reingold AL, Colford JM Jr, et al. Comparison of a whole blood interferon-gamma assay with tuberculin skin testing for the detection of tuberculosis infection in hospitalized children in rural India. Journal of Infection. Mar; 2007 54(3): 267-76. see comment. [PubMed: 16733068]

91. Dominguez J, Ruiz-Manzano J, De Souza-Galvao M, Latorre I, Mila C, Blanco S, et al. Comparison of two commercially available gamma interferon blood tests for immunodiagnosis of tuberculosis. Clin Vaccine Immunol. Jan; 2008 15(1):168-71. [PubMed: 17978008] Comparison of IGRAs in children with active TB ( $n=9)$

92. Hesseling AC, Mandalakas AM, Kirchner LH, Chegou NN, Marais BJ, Zhu X, et al. Highly Discordant T-Cell Responses In Individuals With Recent Household Tuberculosis Exposure. Thorax. Aug 5.2008

93. Nakaoka H, Lawson L, Squire SB, Coulter B, Ravn P, Brock I, et al. Risk for tuberculosis among children. Emerg Infect Dis. Sep; 2006 12(9):1383-8. [PubMed: 17073087]

94. Higuchi K, Harada N, Mori T, Sekiya Y. Use of QuantiFERON((R))-TB Gold to investigate tuberculosis contacts in a high school. Respirology. Jan; 2007 12(1):88-92. [PubMed: 17207031]

95. Connell T, Bar-Zeev N, Curtis N. Early detection of perinatal tuberculosis using a whole blood interferon-gamma release assay. Clin Infect Dis. Jun 1; 2006 42(11):e82-5. [PubMed: 16652300]

96. Richeldi L, Bergamini BM, Vaienti F. Prior tuberculin skin testing does not boost QuantiFERONTB results in paediatric contacts. Eur Respir J. Aug; 2008 32(2):524-5. [PubMed: 18669795]

97. Tsiouris SJ, Austin J, Toro P, Coetzee D, Weyer K, Stein Z, et al. Results of a tuberculosis-specific IFN-gamma assay in children at high risk for tuberculosis infection. Int J Tuberc Lung Dis. Aug; 2006 10(8):939-41. [PubMed: 16898381]

98. Wanchu A, Dong Y, Sethi S, Myneedu VP, Nadas A, Liu Z, et al. Biomarkers for clinical and incipient tuberculosis: performance in a TB-endemic country. PLoS ONE. 2008; 3(4):e2071. [PubMed: 18446210]

99. Walzl G, Ronacher K, Djoba Siawaya JF, Dockrell HM. Biomarkers for TB treatment response: challenges and future strategies. J Infect. Aug; 2008 57(2):103-9. [PubMed: 18649943]

100. Siawaya JF, Bapela NB, Ronacher K, Beyers N, van Helden P, Walzl G. Differential expression of interleukin-4 (IL-4) and IL-4 delta 2 mRNA, but not transforming growth factor beta (TGFbeta), TGF-beta RII, Foxp3, gamma interferon, T-bet, or GATA-3 mRNA, in patients with fast and slow responses to antituberculosis treatment. Clin Vaccine Immunol. Aug; 2008 15(8):116570. [PubMed: 18579694]

101. Kaufmann SH, Parida SK. Tuberculosis in Africa: learning from pathogenesis for biomarker identification. Cell Host Microbe. Sep 11; 2008 4(3):219-28. [PubMed: 18779048]

102. Ruhwald M, Petersen J, Kofoed K, Nakaoka H, Cuevas LE, Lawson L, et al. Improving T-cell assays for the diagnosis of latent TB infection: potential of a diagnostic test based on IP-10. PLoS ONE. 2008; 3(8):e2858. [PubMed: 18682747] *IP-10 is a novel biomarker, expressed in high concentration in response to Mtb antigen and found in children at high risk of infection with Mtb.

103. Locht C, Hougardy JM, Rouanet C, Place S, Mascart F. Heparin-binding hemagglutinin, from an extrapulmonary dissemination factor to a powerful diagnostic and protective antigen against tuberculosis. Tuberculosis (Edinb). May-Jul;2006 86(3-4):303-9. [PubMed: 16510310]

104. Arend SM, Franken WP, Aggerbeck H, Prins C, van Dissel JT, Thierry-Carstensen B, et al. Double-blind randomized Phase I study comparing rdESAT-6 to tuberculin as skin test reagent in the diagnosis of tuberculosis infection. Tuberculosis (Edinb). May; 2008 88(3):249-61. [PubMed: 18155963]

105. Cannas A, Goletti D, Girardi E, Chiacchio T, Calvo L, Cuzzi G, et al. Mycobacterium tuberculosis DNA detection in soluble fraction of urine from pulmonary tuberculosis patients. Int J Tuberc Lung Dis. Feb; 2008 12(2):146-51. [PubMed: 18230246]

106. Keeler E, Perkins MD, Small P, Hanson C, Reed S, Cunningham J, et al. Reducing the global burden of tuberculosis: the contribution of improved diagnostics. Nature. Nov 23; 2006 444(Suppl 1):49-57. [PubMed: 17159894] 


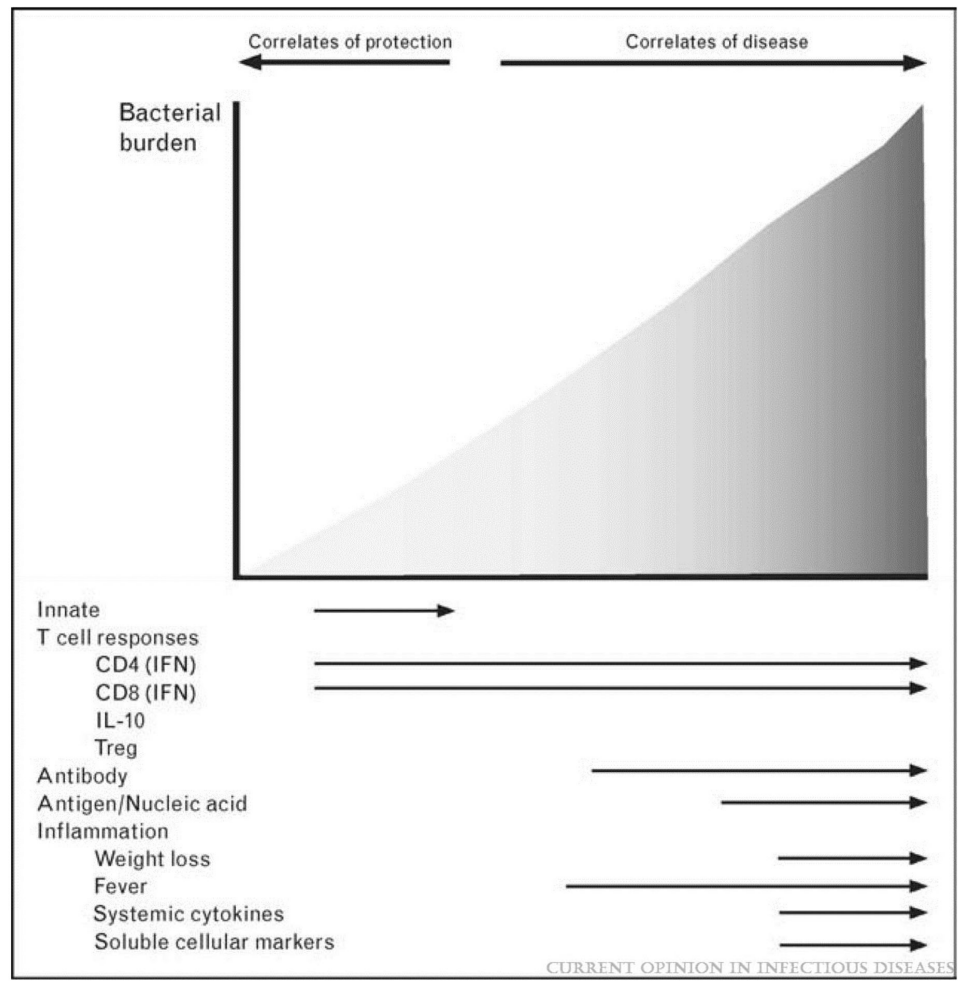

ORIGINAL FIGURE.

This figure illustrates the dynamic interplay involving the host response to infection and diagnosis of Mycobacterium tuberculosis. 